Nonlin. Processes Geophys., 24, 237-253, 2017

https://doi.org/10.5194/npg-24-237-2017

(C) Author(s) 2017. This work is distributed under

the Creative Commons Attribution 3.0 License.

\title{
Ocean swell within the kinetic equation for water waves
}

\author{
Sergei I. Badulin ${ }^{1,2}$ and Vladimir E. Zakharov ${ }^{1,2,3,4,5}$ \\ ${ }^{1}$ P. P. Shirshov Institute of Oceanology of the Russian Academy of Sciences, Moscow, Russia \\ ${ }^{2}$ Laboratory of Nonlinear Wave Processes, Novosibirsk State University, Novosibirsk, Russia \\ ${ }^{3}$ Department of Mathematics, University of Arizona, Tucson, USA \\ ${ }^{4}$ P. N. Lebedev Physical Institute of the Russian Academy of Sciences, Moscow, Russia \\ ${ }^{5}$ Waves and Solitons LLC, Phoenix, Arizona, USA \\ Correspondence to: Sergei I. Badulin (badulin.si@ ocean.ru)
}

Received: 6 October 2016 - Discussion started: 17 October 2016

Revised: 26 April 2017 - Accepted: 29 April 2017 - Published: 6 June 2017

\begin{abstract}
Results of extensive simulations of swell evolution within the duration-limited setup for the kinetic Hasselmann equation for long durations of up to $2 \times 10^{6} \mathrm{~s}$ are presented. Basic solutions of the theory of weak turbulence, the so-called Kolmogorov-Zakharov solutions, are shown to be relevant to the results of the simulations. Features of selfsimilarity of wave spectra are detailed and their impact on methods of ocean swell monitoring is discussed. Essential drop in wave energy (wave height) due to wave-wave interactions is found at the initial stages of swell evolution (on the order of $1000 \mathrm{~km}$ for typical parameters of the ocean swell). At longer times, wave-wave interactions are responsible for a universal angular distribution of wave spectra in a wide range of initial conditions. Weak power-law attenuation of swell within the Hasselmann equation is not consistent with results of ocean swell tracking from satellite altimetry and SAR (synthetic aperture radar) data. At the same time, the relatively fast weakening of wave-wave interactions makes the swell evolution sensitive to other effects. In particular, as shown, coupling with locally generated wind waves can force the swell to grow in relatively light winds.
\end{abstract}

\section{Physical models of ocean swell}

Ocean swell is an important constituent of the field of surface gravity waves in the sea and, more generally, of the sea environment as a whole. Swell is usually defined as a fraction of a wave field that does not depend (or depends slightly) on local wind. Being generated in confined stormy areas, these waves can propagate long distances of many thousands of miles, thus influencing vast ocean stretches. For example, swell from the Roaring Forties in the Southern Ocean can traverse the Pacific and reach distant shores of California and Kamchatka. Predicting swell as a part of sea wave forecast remains a burning problem for maritime safety and marine engineering.

Pioneering works by Barber and Ursell (1948), Munk et al. (1963), and Snodgrass et al. (1966) discovered a rich physics of the phenomenon and gave the first examples of accurate measurements of magnitudes, periods and directional spreading of swell. All the articles contain thorough discussions of the physical background of swell generation, attenuation and interaction with other types of ocean motions. A fascinating story of a grand experiment on ocean swell has been presented to a wide audience in the documentary "Waves across the Pacific" (can be found at https: $/ /$ www.youtube.com/watch?v=MX5cKoOm6Pk). ${ }^{1}$

Nonlinear wave-wave interactions have been sketched by Snodgrass et al. (1966) as a novelty introduced by the milestone papers by Phillips (1960) and Hasselmann (1962). A possible important role of these interactions at high swells for relatively short times of evolution has been outlined and evaluated. The first estimates of the observed rates of swell attenuation were carried out by Snodgrass et al. (1966) based on observation at near-shore stations. Their $e$-folding scale of about $4000 \mathrm{~km}$ (the distance at which an exponentially decaying wave height decreases by a factor of $e$ ) is consistent with some of today's results of the satellite tracking of swell (Ardhuin et al., 2009, 2010; Jiang et al., 2016) and with the

\footnotetext{
${ }^{1}$ The authors are thankful to Gerbrant van Vledder, Delft University of Technology, for this reference.
} 
treatment of these results within the model of swell attenuation due to coupling with the turbulent atmospheric layer (e.g., Tsimring, 1986; Kantha, 2006). The alternative semiempirical model of Babanin (2006) predicts a quite different algebraic law and stronger swell attenuation at shorter distances from the swell source (Young et al., 2013). Note that the effect of the decay of a monochromatic wave due to turbulent wave flow is found to be quadratic in wave amplitude, i.e., to be of lower-order nonlinearity than in the nondissipative theory of weakly nonlinear water waves.

It should be stressed that a number of theoretical and numerical models, including those mentioned above, treat swell as a quasi-monochromatic wave and, thus, ignore nonlinear interactions of the swell harmonics themselves and the swell coupling with locally generated wind waves. The latter effect can be essential, as observations and simulations clearly show (e.g., Kahma and Pettersson, 1994; Pettersson, 2004; Young, 2006; Badulin et al., 2008b, and references therein). Usually the swell continues to be considered a superposition of harmonics that do not interact with each other and, thus, can be described by the well-known methods of the linear theory of waves (e.g., Ewans, 1998; Ewans et al., 2004). Many features of the observed swell can be related to such models. For example, the observed effect of linear growth of the swell frequency at a site can be explained as an effect of dispersion of a linear wave packet over a long time and successfully used for relating these observations to stormy areas that generate the swell (e.g., Barber and Ursell, 1948; Ewans et al., 2004).

Synthetic aperture radar (SAR) allows for a spatial resolution of up to tens of meters (e.g., Ardhuin et al., 2010; Young et al., 2013). Satellite altimeters measure wave height averaged over a snapshot of a few square kilometers. These snapshots are adequate for currently known methods of statistical description of waves in research and application models. These can be used for swell tracking in combination with other tools (e.g., wave models as in Jiang et al., 2016). Retracking of swells allows us, first, to relate the swell events to their probable sources - stormy areas - and, secondly, the swell transformation enables us to estimate the effects of other motions of the atmosphere and ocean - seasonal wind activity (e.g., Chen et al., 2002), wave-current interaction (e.g., Beal et al., 1997) and bathymetry effects (Young et al., 2013). Such a work requires adequate physical models of swell propagation and transformation. This paper aims to narrow the gap.

Meanwhile, the linear treatment remains quite restrictive and cannot explain important features of swell. The observed swell spectra exhibit frequency downshift which is not predicted by deterministic linear or weakly nonlinear models of narrow-banded wave guide evolution (e.g., data of Snodgrass et al., 1966, and comments on these data by Henderson and Segur, 2013). Moreover, these spectra show invariance of their shapes that is unlikely to appear in a linear dispersive wave system. These noted features are common for wave spectra described by the kinetic equation for water waves, the so-called Hasselmann (1962) equation.

In this paper we present results of extensive simulations of ocean swell within the Hasselmann equation for deep water waves. The simplest duration-limited setup has been chosen to obtain numerical solutions for the duration of up to $2 \times 10^{6} \mathrm{~s}$ (about 23 days) for typical parameters of ocean swell (wavelengths $150-400 \mathrm{~m}$, wave periods $10-16 \mathrm{~s}$, initial significant heights 3-15 m).

We analyze the simulation results within the framework of the theory of weak turbulence (Zakharov et al., 1992). The slowly evolving swell solutions appear to be quite close to the stationary Kolmogorov-Zakharov spectra. We give a short theoretical introduction and present estimates of the basic constants of the theory in the next section. In Sect. 3 we relate results of simulations to properties of the self-similar solutions of the kinetic equation. Zaslavskii (2000) was the first to present the self-similar solutions for swell assuming the angular narrowness of the swell spectra and stated explicit analytical results. In fact, more general consideration, in the spirit of Badulin et al. (2002, 2005a), leads to important findings and raises questions independently of the assumption of angular narrowness.

We demonstrate the fact that is usually ignored: the powerlaw swell attenuation within the conservative kinetic equation. We show that it does not contradict the observations mentioned above. We also reveal a remarkable feature of collapsing the swell spectra onto an angular distribution that depends weakly on initial angular spreading. Such universality can be of great value for modeling swell and developing methods for its monitoring (Delpey et al., 2010).

We conclude this paper with a discussion of how to apply this model. Evidently, the setup of duration-limited evolution is quite restrictive and does not reflect essential features of ocean swell when wave dispersion and spatial divergence play a key role. At the same time, wave-wave interactions remain of importance independently of the setup. The weakening of swell evolution is not directly related to abatement of wave-wave interactions which are able to effectively restore perturbations of these quasi-stationary states (Zakharov and Badulin, 2011). On the contrary, this favors coupling of the quasi-stationary swell with the ocean environment. In particular, the locally generated wind-driven waves can switch the swell attenuation to swell amplification. This effect can be considered for interpretation of recent observations of swell from space ("negative" dissipation in the words of Jiang et al., 2016). Many problems of adequate physical description of swell in the ocean are still open. This paper is an attempt to reveal essential features of swell evolution within the simplest model of the kinetic Hasselmann equation. 


\section{Solutions for ocean swell}

\subsection{The Kolmogorov-Zakharov solutions}

In this section we reproduce previously reported theoretical results on the evolution of swell as a random field of weakly interacting wave harmonics. We apply the statistical theory of wind-driven seas (Zakharov, 1999) to the sea swell, whose description with this approach is usually considered questionable. A random wave field is described by the kinetic equation derived by Hasselmann (1962) for weakly nonlinear deep water waves in the absence of dissipation and external forcing:

$\frac{\partial N_{k}}{\partial t}+\nabla_{\boldsymbol{k}} \omega_{\boldsymbol{k}} \nabla_{\boldsymbol{r}} N_{\boldsymbol{k}}=S_{\mathrm{nl}}$.

Equation (1) is written for the spectral density of wave action $N(\boldsymbol{k}, \boldsymbol{x}, t)=E(\boldsymbol{k}, \boldsymbol{x}, t) / \omega(\boldsymbol{k})(E(\boldsymbol{k}, \boldsymbol{x}, \boldsymbol{t})$ is the wave energy spectrum and the wave frequency obeys the linear dispersion relation $\omega=\sqrt{g|\boldsymbol{k}|})$. Subscripts for $\nabla$ correspond to the twodimensional gradient operator in the corresponding space of coordinates $\boldsymbol{x}$ and wavevectors $\boldsymbol{k}$ (i.e., $\left.\nabla_{\boldsymbol{r}}=(\partial / \partial x, \partial / \partial y)\right)$.

The right-hand term $S_{\mathrm{nl}}$ describes the effect of wave-wave resonant interactions and can be written in an explicit form (see the Appendices in Badulin et al., 2005a, for a collection of the formulas). The cumbersome term $S_{\mathrm{nl}}$ causes many problems for wave modeling whenever Eq. (1) is extensively used. Nevertheless, for the deep water case, one has a key property of homogeneity

$S_{\mathrm{nl}}\left[\kappa \boldsymbol{k}, v N_{\boldsymbol{k}}\right]=\kappa^{19 / 2} v^{3} S_{\mathrm{nl}}\left[\boldsymbol{k}, N_{\boldsymbol{k}}\right]$

that helps in acquiring important analytical results. Stretching in $\kappa$ times in the wave scale or in $v$ times in the wave action, where $\kappa, v$ are positive, leads to simple re-scaling of the collision term, $S_{\mathrm{nl}}$. This important property gives a clue for constructing power-law stationary solutions of the kinetic equation, i.e., solutions for the equation

$S_{\mathrm{nl}}=0$.

Two isotropic stationary solutions of Eq. (3) correspond to constant fluxes of wave energy and action in wave scales. The direct cascade solution (Zakharov and Filonenko, 1966) in terms of the frequency spectrum of energy

$E^{(1)}(\omega, \theta)=2 C_{p} \frac{P^{1 / 3} g^{4 / 3}}{\omega^{4}}$

introduces the basic Kolmogorov constant $C_{p}$ and describes the energy transfer to infinitely short waves with constant flux $P$. The wave action transfer in the opposite direction of long waves is described by the inverse cascade solution (Zakharov and Zaslavsky, 1982) with wave action flux $Q$ and another Kolmogorov constant $C_{q}$ :

$E^{(2)}(\omega, \theta)=2 C_{q} \frac{Q^{1 / 3} g^{4 / 3}}{\omega^{11 / 3}}$.
Note that key features of the isotropic KolmogorovZakharov solution Eqs. (4) and (5) are reproduced quite well by means of direct numerical simulations based on the integro-differential Zakharov equation (Annenkov and Shrira, 2006) or on the primitive Euler equations (Onorato et al., 2002).

An approximate weakly anisotropic KolmogorovZakharov solution has been obtained by Kats and Kontorovich (1974) as an extension of Eq. (4):

$E^{(3)}(\omega, \theta)=2 \frac{P^{1 / 3} g^{4 / 3}}{\omega^{4}}\left(C_{p}+C_{m} \frac{g M}{\omega P} \cos \theta+\ldots\right)$.

It associates the wave spectrum anisotropy with the constant spectral flux of wave momentum $M$ and the so-called second Kolmogorov constant $C_{m}$. As is seen from Eq. (6), the solution anisotropy vanishes as $\omega \rightarrow \infty$ : wave spectra become isotropic for short waves. The whole set of the KolmogorovZakharov (KZ) solutions (Eqs. 4-6) can be treated naturally within the dimensional approach: these are just particular cases of solutions of the form

$E^{(K Z)}(\omega)=\frac{P^{1 / 3} g^{4 / 3}}{\omega^{4}} G(\omega Q / P, g M /(\omega P), \theta)$,

where $G$ is a function of dimensionless arguments scaled by spectral fluxes of wave energy $P$, action $Q$ and momentum $M$.

Originally, solutions (Eqs. 4-6) were derived in particularly sophisticated and cumbersome ways. Later on, simpler and more physically transparent approaches were presented (Zakharov and Pushkarev, 1999; Balk, 2000; Pushkarev et al., 2003, 2004; Badulin et al., 2005a; Zakharov, 2010). These more general approaches allow us to find higher-order terms of the anisotropic Kolmogorov-Zakharov solutions (Eq. 6). In particular, they predict the next term to be proportional to $\cos 2 \theta / \omega^{2}$, which is the second angular harmonics of the stationary solution (Eq. 6).

Swell solutions evolve slowly with time and, thus, give a good opportunity for discussing features of the $\mathrm{KZ}$ solutions (or, alternatively, the $\mathrm{KZ}$ solutions can be used as a reference case for the swell studies). One of the key points of this discussion is the question of uniqueness or universality of the swell solutions that can be treated in the context of general KZ solutions (Eq. 7). The principal terms of the general Kolmogorov-Zakharov solutions (Eqs. 4-6) have the clear physical meanings of total fluxes of wave action, Eq. (5), energy, Eq. (4), and momentum, Eq. (6), and do not refer to specific initial conditions. This is not the case for the higherorder terms. The link between these additional terms with inherent properties of the collision integral $S_{\mathrm{nl}}$ and/or with specific initial conditions is a subject of further studies.

\subsection{Self-similar solutions of the kinetic equation}

The homogeneity property Eq. (2) is extremely useful for studies of non-stationary (inhomogeneous) solutions of the 
kinetic equation. Approximate self-similar solutions for reference cases of duration- and fetch-limited development of the wave field can be obtained under the assumption of dominance of the wave-wave interaction term $S_{\mathrm{nl}}$ (Pushkarev et al., 2003; Zakharov, 2005; Badulin et al., 2005a; Zakharov and Badulin, 2011). These solutions exhibit the so-called incomplete or second-type self-similarity (e.g., Barrenblatt, 1979). In terms of frequency-angle dependencies of wave action spectra, one has correspondingly for the duration- and fetch-limited cases (Badulin et al., 2005a, 2007; Zakharov et al., 2015)

$N(\omega, \theta, \tau)=a_{\tau} \tau^{p_{\tau}} \Phi_{p_{\tau}}(\xi, \theta)$,

$N(\omega, \theta, \chi)=a_{\chi} \chi^{p_{\chi}} \Phi_{p_{\chi}}(\zeta, \theta)$,

with dimensionless time $\tau$ and fetch $\chi$ :

$\tau=t / t_{0} ; \quad \chi=x / x_{0}$.

Dimensionless arguments of shape functions $\Phi_{p_{\tau}}(\xi)$ and $\Phi_{p_{\chi}}(\zeta)$ in Eqs. (8) and (9) contain free scaling parameters $b_{\tau}$ and $b_{\chi}$ and exponents of frequency downshifting $q_{\tau}$ and $q_{\chi}$ :

$\xi=b_{\tau} \omega^{2} \tau^{-2 q_{\tau}} ; \quad \zeta=b_{\chi} \omega^{2} \chi^{-2 q_{\chi}}$.

The homogeneity property (Eq. 2) dictates "magic relations" (in the words of Pushkarev and Zakharov, 2015, 2016) between exponents $p_{\tau}, q_{\tau}$ and $p_{\chi}, q_{\chi}$

$p_{\tau}=\frac{9 q_{\tau}-1}{2} ; \quad p_{\chi}=\frac{10 q_{\chi}-1}{2}$.

Additional "magic relations" coming from the homogeneity property (Eq. 2) fix a link between the amplitude scales $a_{\tau}, a_{\chi}$ and the bandwidth scales $b_{\tau}, b_{\chi}$ of the self-similar solutions (Eqs. 8-11):

$a_{\tau}=b_{\tau}^{19 / 4} ; \quad a_{\chi}=b_{\chi}^{5 / 2}$.

Thus, "magic relations" (Eqs. 12 and 13) reduce the number of free parameters of the self-similar solutions (Eqs. 8 and 9) from four (two exponents and two coefficients) to only two: a dimensionless exponent $p_{\tau}\left(p_{\chi}\right)$ and an amplitude of the solution $a_{\tau}\left(a_{\chi}\right)$.

The shape functions $\Phi_{p_{\tau}}(\xi, \theta)$ and $\Phi_{p_{\chi}}(\zeta, \theta)$ in Eqs. (8) and (9) are specified by solutions of a nonlinear boundary problem for an integro-differential equation in self-similar variables $\xi$ or $\zeta$ (conditions of decay at zero and infinity) and angle $\theta$ (periodicity) (see Sect. 5.2 in Badulin et al., 2005a, for details). These solutions reveal relatively narrow angular distributions with a single pronounced maximum and remarkably weak dependence on the exponent of wave growth $p_{\xi},\left(p_{\chi}\right)$, as simulations show (e.g., Badulin et al., 2008a). This feature of quasi-universality (in the words of Badulin et al., 2005a) of the solutions of a nonlinear problem can be treated within a diffusion approximation for the kinetic equation (Zakharov and Pushkarev, 1999, and Zakharov, 2010) as a "survival" of very few eigenfunctions - angular harmonics of the corresponding linear boundary problem. As will be shown below, the weakly anisotropic KZ solution (Eq. 6) represents a principal angular harmonic of such a decomposition.

Two-lobe patterns can be observed beyond the spectral peak as local maxima at oblique directions or as "shoulders" in wave frequency spectra. Their appearance within the kinetic equation approach is generally associated with wave generation by wind (e.g., Bottema and van Vledder, 2008, 2009) and/or the effect of wave-wave interactions (Banner and Young, 1994; Pushkarev et al., 2003). Numerical simulations within the potential Euler equations also show formation of the two-lobe patterns for rather short times (a few hundred spectral peak periods) of the evolution of an initially unimodal spectral distribution (Toffoli et al., 2010).

An essential approximation which is widely used both for experimentally observed and simulated wave spectra is generally treated as an important property of spectral shape invariance (terminology of Hasselmann et al., 1976) or the spectra quasi-universality (in the words of Badulin et al., 2005a). In fact, such "invariance" does not suppose a pointby-point matching of properly normalized spectral shapes. The proximity of integrals of the shape functions $\Phi_{p_{\tau}}, \Phi_{p_{\chi}}$ in a range of wave growth rates $p_{\tau}, p_{\chi}$ appears to be sufficient, in particular, for formulating efficient semi-empirical parameterizations of wind-wave growth in terms of integral values (e.g., Hasselmann et al., 1976). Consistent analysis within the weak turbulence approach that used this important approximation has recently lead to a remarkable theoretically based relationship (Zakharov et al., 2015):

$\mu^{4} v=\alpha_{0}^{3}$.

Here wave steepness $\mu$ is estimated from total wave energy $E$ and spectral peak frequency $\omega_{\mathrm{p}}$ :

$\mu=\frac{E^{1 / 2} \omega_{\mathrm{p}}^{2}}{g}$.

The "number of waves" $v$ in a spatially homogeneous wind sea (i.e., for the duration-limited case) is defined as follows:

$v=\omega_{\mathrm{p}} t$.

For spatial (fetch-limited) wave growth, the coefficient of proportionality $C_{f}$ in the equivalent expression $v=C_{f}\left|\boldsymbol{k}_{p}\right| x$ ( $\boldsymbol{k}_{p}$ being the wavevector of the spectral peak) is close to the ratio between the phase and group velocities $C_{p h} / C_{g}=2$. A universal constant $\alpha_{0} \approx 0.7$ is a counterpart of the constants $C_{p}, C_{q}$ of the stationary Kolmogorov-Zakharov solutions (Eqs. 4 and 5) and has a similar physical meaning of a ratio between wave energy and the energy spectral flux (in power 1/3). A remarkable feature of the universal wave growth law (Eq. 14) is its independence of wind speed. This wind-free paradigm based on intrinsic scaling of wave development is shown to be a useful tool of analysis of wind-wave 
growth (Zakharov et al., 2015). Below we demonstrate its effectiveness for interpreting swell simulations.

\subsection{Self-similarity of swell solutions}

The self-similar solution for swell is just a member of a family of solutions (Eqs. 8 and 9) with special values of temporal or spatial rates:

$p_{\tau}=1 / 11 ; \quad q_{\tau}=1 / 11$,

$p_{\chi}=1 / 12 ; \quad q_{\chi}=1 / 12$.

Exponents (Eqs. 17 and 18) provide conservation of the total wave action for its evolution in time (duration-limited setup) or in space (fetch-limited):

$N=\int_{0}^{+\infty} \int_{-\pi}^{+\pi} N(\omega, \theta) \mathrm{d} \omega \mathrm{d} \theta=$ const.

By contrast, total energy

$E=\int \omega N(\boldsymbol{k}) \mathrm{d} \boldsymbol{k}$

and wave momentum

$\mathbf{K}=\int \boldsymbol{k} N(\boldsymbol{k}) \mathrm{d} \boldsymbol{k}$

are only formal constants of motion of the Hasselmann equation and decay with time $t$ or fetch $x$

$E \sim t^{-1 / 11} ; \quad K_{x} \sim t^{-2 / 11}$,

$E \sim x^{-1 / 12} ; \quad K_{x} \sim x^{-2 / 12}$.

The swell decay (Eqs. 22 and 23) reflects a basic feature of the kinetic equation for water waves: energy Eq. (20) and momentum Eq. (21) are not conserved (see Zakharov et al., 1992; Pushkarev et al., 2003, and references herein). The wave action is the only true integral of the kinetic Eq. (1).

The swell solution manifests another general feature of evolving spectra: the downshifting of the spectral peak frequency (or other characteristic frequency), i.e.,

$\omega_{\mathrm{p}} \sim t^{-1 / 11} ; \quad \omega_{\mathrm{p}} \sim x^{-1 / 12}$.

The universal law of wave evolution (Eq. 14) is, evidently, valid for the self-similar swell solution as well with a minor difference in the value of the constant $\alpha_{0}$. As soon as this constant is expressed in terms of the integrals of the shape functions $\Phi_{p_{\tau}}, \Phi_{p_{\chi}}$ and the swell spectrum shape differs essentially from ones of the growing wind seas, this constant appears to be less than $\alpha_{0}$ of the growing wind seas.

The theoretical background presented above is used below for analysis of results of simulations.

\section{Swell simulations}

\subsection{Simulation setup}

Simulations of ocean swell require special care. First of all, calculations for quite long periods of time (up to $2 \times 10^{6} \mathrm{~s}$ in our case) should be accurate enough in order to capture relatively slow evolution of solutions and, thus, be able to relate results to the theoretical background presented above. Duration-limited evolution of the swell has been simulated with the Pushkarev et al. (2003) version of the code based on the WRT algorithm (Webb, 1978; Tracy and Resio, 1982). Features of the code and numerical setups have been described in previous papers (Badulin et al., 2002, 2004, 2005a, b, 2007; Zakharov et al., 2007; Badulin et al., 2008a, 2013; Pushkarev and Zakharov, 2015, 2016). The frequency resolution for log-spaced grid has been set to $\left(\omega_{n+1}-\omega_{n}\right) / \omega_{n}=$ 1.03128266. It corresponds to 128 grid points in the frequency range $0.02-1 \mathrm{~Hz}$ (approximately 1.5 to $3850 \mathrm{~m}$ wavelength).

Standard angular resolution $\Delta \theta=10^{\circ}$ has been taken as adequate for the goals of our study. A control series of runs with angular resolution $\Delta \theta=5^{\circ}$ showed very close but still quantitatively different shaping of wave spectra (see discussion below), while differences in integral parameters (wave height, period, total momentum) did not exceed $1 \%$ after $2 \times 10^{6}$ s of evolution.

Initial conditions were similar in all series of simulations: spectral density of action in wavenumber space was almost constant in a box of the wavenumber modulo and angles. A slight modulation ( $5 \%$ of the box height) and a low pedestal outside the box ( 6 orders less than the maximal value) have been set in order to stimulate wave-wave interactions since the collision integral $S_{\mathrm{nl}}$ vanishes for $N(\boldsymbol{k})=$ const:

$N(\boldsymbol{k})=\left\{\begin{array}{l}N_{0}\left(1+0.05 \cos ^{2}(\theta / 2)\right),|\theta|<\Theta / 2, \omega_{l}<\omega<\omega_{h} \\ 10^{-6} N_{0}, \quad \text { otherwise. }\end{array}\right.$

In Eq. (25) the references to angle $\theta\left(\cos \theta=k_{x} /|\boldsymbol{k}|\right)$ and wave frequency $\omega$ are used for conciseness of the expression for spatial wave action spectrum $N(\boldsymbol{k})$. The default values $\omega_{l}$ and $\omega_{h}$ corresponding to wave periods 10 and $2.5 \mathrm{~s}$ have been used for the most cases providing sufficient space for spectral evolution to low frequencies (spectra downshifting) and for stability of calculations at high frequencies for the default cutoff frequency $f_{\mathrm{c}}=1 \mathrm{~Hz}$.

Dissipation was absent in the runs. Free boundary conditions were applied at the high-frequency end of the domain of calculations: generally, short-term oscillations of the spectrum tail do not lead to instability; i.e., the resulting solutions can be regarded as ones corresponding to conditions of decay at infinitely small scales $(N(\boldsymbol{k}) \rightarrow 0$ when $|\boldsymbol{k}| \rightarrow \infty)$.

Calculations with a hyper-viscosity (e.g., Pushkarev et al., 2003) or a diagnostic tail at the high-frequency range of the spectrum (Gagnaire-Renou et al., 2010) do not affect results quantitatively compared to our simulations without any dis- 
Table 1. Initial parameters of the simulation series.

\begin{tabular}{lrrr}
\hline ID & $\Theta$ & $N\left(\mathrm{~m}^{2} \mathrm{~s}\right)$ & $H_{S}(\mathrm{~m})$ \\
\hline sw030 & $30^{\circ}$ & 0.720 & 4.63 \\
sw050 & $60^{\circ}$ & 0.719 & 4.6 \\
sw170 & $180^{\circ}$ & 0.714 & 4.74 \\
sw230 & $240^{\circ}$ & 0.721 & 4.67 \\
sw330 & $330^{\circ}$ & 0.722 & 4.79 \\
\hline
\end{tabular}

sipation. Thus, these "non-conservative" options can mimic successfully the effect of energy leakage at $|\boldsymbol{k}| \rightarrow \infty$ in our formally non-dissipative problem. Very strong dissipation at less than 10 grid points at the very end of frequency domain suppresses the spectral level and, simultaneously, reduces the overall energy dissipation at these points. Thus, the effect on the evolution of the energy-containing part of the solution appears to be quite weak and depends slightly on the particular form and magnitude of the hyper-viscosity. In some cases, the hyper-viscosity option that suppresses high-frequency noise can accelerate calculations. In a sense, it is equivalent to reducing an effective number of grid points. Test runs with the reduced frequency domain (cutoff up to $f_{\mathrm{c}}=0.6 \mathrm{~Hz}, 112$ grid points) did not show an essential quantitative difference with the default option $\left(f_{\mathrm{c}}=1 \mathrm{~Hz}, 128\right.$ grid points).

In contrast to wind-driven waves where wind speed is an essential physical parameter that gives a useful physical scale, the swell evolution is determined by initial conditions only, i.e., by $N_{0}$ (dimension of wave action spectral density $[N(\boldsymbol{k})]=\left[\right.$ Length $^{4}$. Time $]$ ), a characteristic frequency (sideband $\left.\left[\omega_{l}, \omega_{h}\right]\right)$ and angular spreading $\Theta$ within the setup (Eq. 25). We tried different combinations of these parameters. Three frequency bands [0.026-0.09], [0.058-0.25], and $[0.1-0.4] \mathrm{Hz}$ have been chosen to generate swell with wavelengths approximately 200,300 , and $400 \mathrm{~m}$ at the final stages of evolution. The angular spreading $\Theta$ was set at 30, 50, 170, 230 and $330^{\circ}$. Initial significant wave heights $H_{S}$ were taken as approximately $4.8,8,10,12$, and $18 \mathrm{~m}$. As will be detailed below, an abrupt fall in wave energy occurred at the very first hours of evolution (up to $50 \%$ for the first $1 \mathrm{~h}$ ). Thus, the above high values of $H_{S}$ can be accepted as realistic values for sea swell. In total, more than 30 combinations of wave height, frequency range and angular spreading have been simulated for the duration of at least $10^{6} \mathrm{~s}$. In some cases, for high amplitudes and narrow angular spreadings, simulations have failed because of strong numerical instability.

Below we focus on the series of Table 1 where initial wave heights were fixed (within 2\%) at approximately $4.8 \mathrm{~m}$ and angular spreading varied from very narrow, $\Theta=30^{\circ}$, to almost isotropic, $\Theta=330^{\circ}$ (Eq. 25). The frequency range of the initial perturbations was $0.1-0.4 \mathrm{~Hz}$. The simulations have been carried out for duration $2 \times 10^{6} \mathrm{~s}$ with angular res-

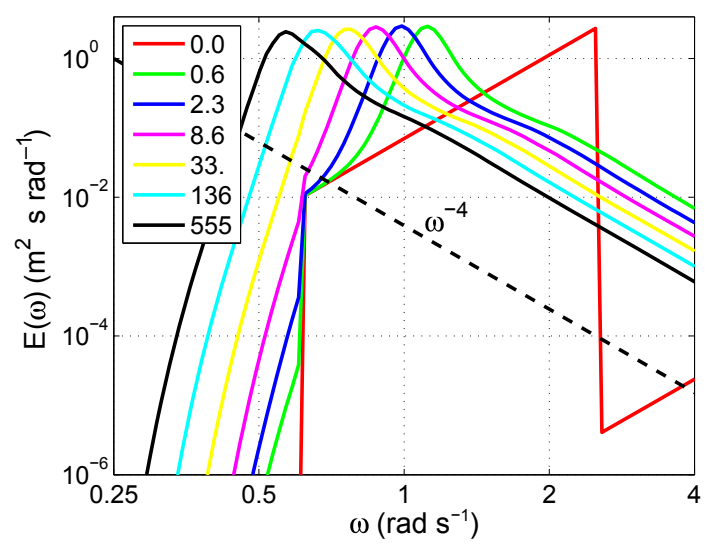

Figure 1. Frequency spectra of energy at different times (legend, in hours) for case $\operatorname{sw} 330\left(\Theta=330^{\circ}\right)$.

olution $\Delta \theta=10^{\circ}$ and checked for series sw030 and sw330 with $\Delta \theta=5^{\circ}$.

\subsection{Self-similar features of swell}

Evolution of swell spectra with time is shown in Fig. 1 for case sw330 of Table 1 . The example shows a strong tendency to self-similar shaping of wave spectra. This remarkable feature has been demonstrated and discussed for swell in previous works (Badulin et al., 2005a; Benoit and GagnaireRenou, 2007; Gagnaire-Renou et al., 2010) for special parameters that provided relatively fast evolution of rather short and unrealistically high waves. In our simulations, we start with the mean wave period of about $3 \mathrm{~s}$ that corresponds to the end of calculations of Badulin et al. (2005a, see Fig. 8 therein). The initial spectrum evolves very quickly and keeps a characteristic shape for less than $1 \mathrm{~h}$, when wave steepness falls dramatically below $\mu=0.15\left(T_{p} \approx 6 \mathrm{~s}\right)$, while wave height loses only about $20 \%$ of its initial value (see Fig. 1, green curve for $t=0.6 \mathrm{~h}$ ). For $555 \mathrm{~h}$, the spectral peak period reaches $11.4 \mathrm{~s}$ (the corresponding wavelength $\lambda \approx 200 \mathrm{~m}$ ) and wave steepness becomes $\mu=0.022$. The final significant wave height $H_{s} \approx 2.8 \mathrm{~m}$ is essentially less than its initial value $4.8 \mathrm{~m}$. All these values can be considered typical ones for ocean swell.

The dependence of key wave parameters on time is shown in Fig. 2 for different runs of Table 1. Power-law dependencies of self-similar solutions (Eqs. 17, 18 and 22-24) are shown by dashed lines. In Fig. 2a, b total wave energy $E$ and the spectral peak frequency $\omega_{\mathrm{p}}$ show good correspondence to power laws of the self-similar solutions (Eq. 8). By contrast, the power-law decay of the $x$-component of wave momentum $K_{x}$ depends essentially on angular spreading of initial wave spectra. While for narrow spreading (runs sw030 and sw050) there is no visible deviation from the $K_{x} \sim t^{-2 / 11}$ law, wide-angle cases clearly show these deviations. The "almost isotropic" solution for sw330 tends quite slowly to the theoretical dependency of wave momentum $K_{x}$ (Eq. 23). The 
(a)

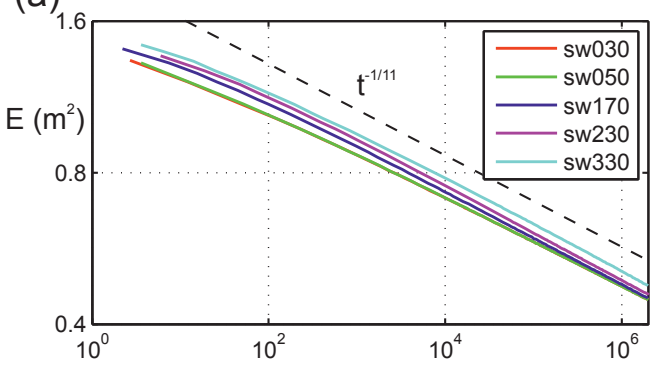

(c)

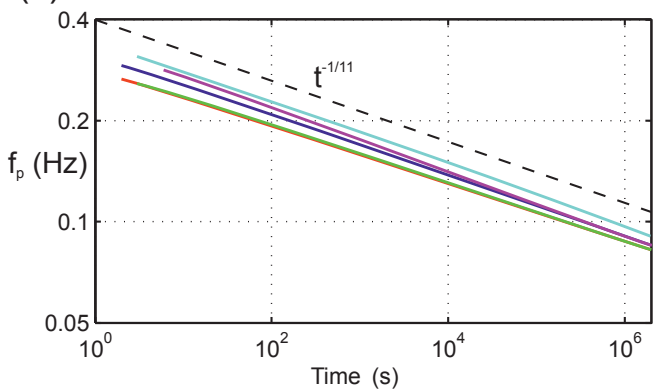

(b)

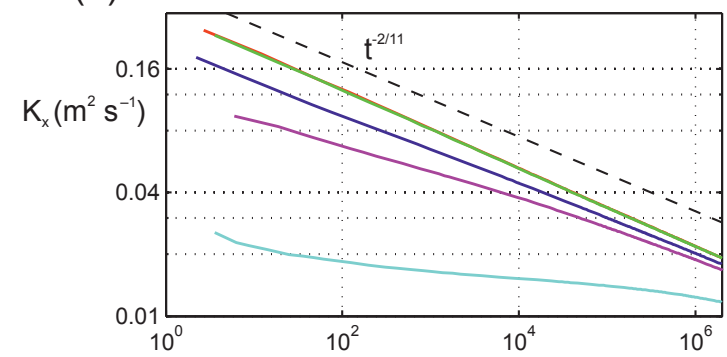

(d)

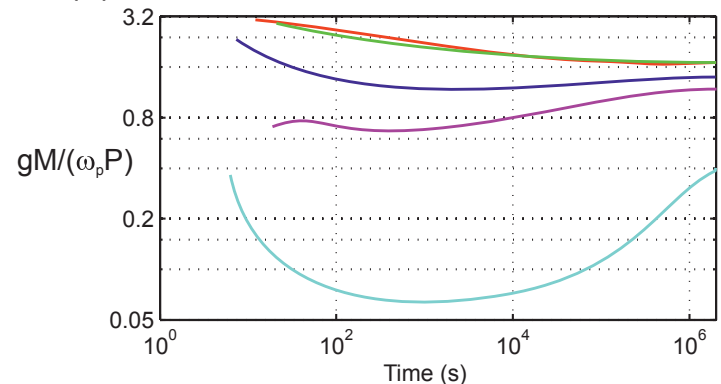

Figure 2. Evolution of wave parameters for runs of Table 1 (in the legend): (a) total energy $E$; (b) total wave momentum $K_{x}$; (c) frequency $f_{p}=\omega_{\mathrm{p}} /(2 \pi)$ of the energy spectra peak; (d) estimate of the parameter of anisotropy $A$ (Eq. 26). Dashed lines show asymptotic power laws (Eqs. 22 and 24)

duration of more than 3 weeks appears "too short": one can see a transitional behavior when wave spectra evolve from the "almost isotropic" state to an inherent distribution with a pronounced anisotropy.

A simple quantitative estimate of the "degree of anisotropy" is given in Fig. 2d. Evolution of the dimensionless parameter of anisotropy in terms of the approximate Kolmogorov-Zakharov solution (Eq. 6) by Kats and Kontorovich (1974) is shown for all the cases of Table 1. We introduce the parameter of anisotropy $A$ as follows:

$A=\frac{g M}{\omega_{\mathrm{p}} P}$,

where the total energy flux $P$ (energy flux at $\omega \rightarrow \infty$ ) is estimated from the evolution of total energy:

$P=-\frac{\mathrm{d} E}{\mathrm{~d} t}$.

Similarly, the total wave momentum (Eq. 21) provides an estimate of its flux as follows:

$M=-\frac{\mathrm{d} K_{x}}{\mathrm{~d} t}$.

Spectral peak frequency $\omega_{\mathrm{p}}$ has been used for the definition of "degree of anisotropy", $A$ (Eq. 26). Different scenarios are seen in Fig. 2d depending on angular spreading of wave spectra. Nevertheless, a general tendency to a universal behavior at very large times (more than $2 \times 10^{6} \mathrm{~s}$ ) looks quite plausible.

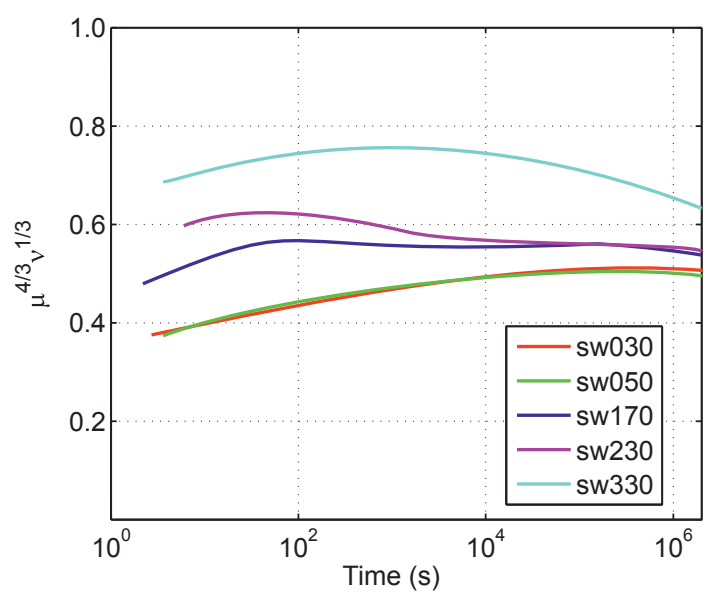

Figure 3. Evolution of the left-hand side of the invariant (Eq. 14) $\left(\mu^{4} v\right)^{1 / 3}$ for runs of Table 1 (in the legend).

Similar dispersion of runs depending on anisotropy of initial distributions is seen in Fig. 3 when tracing the invariant of the self-similar solutions (Eq. 14). Again, like in Fig. 2b, $2 \times 10^{6} \mathrm{~s}$ are not sufficient to demonstrate the validity of a relationship (Eq. 14) in full. A limit $\alpha_{0}$ (Eq. 14) is very likely reached at larger times. This limit is a bit less (by approximately $15 \%$ ) than one for growing wind seas: $\alpha_{0} \approx 0.7$. Again, the "almost isotropic" solution shows its stronger departure from the rest of the series. The differences are better seen in angular distributions rather than in normalized 


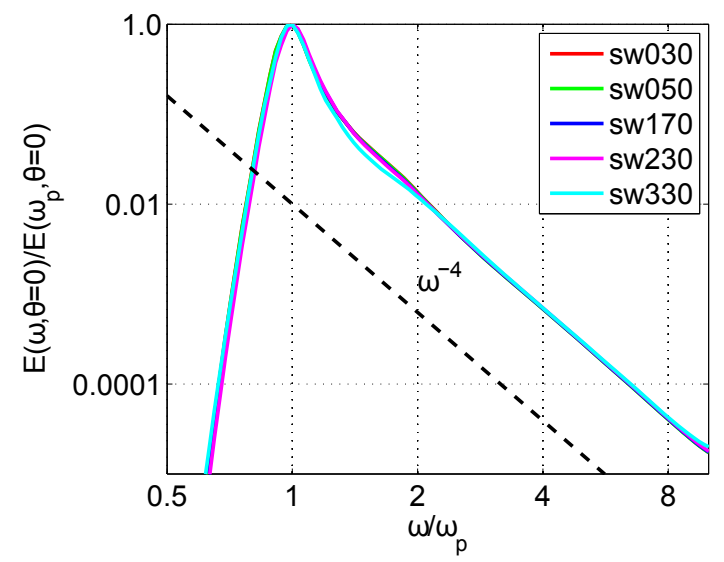

Figure 4. Normalized frequency spectra for direction $\theta=0^{\circ}$ after 11.5 days (approximately $10^{6} \mathrm{~s}$ ) of swell evolution for runs of Table 1 (see the legend).

spectral shapes (Fig. 4) when we are trying to check selfsimilarity features of the solutions in the spirit of Badulin et al. (2005a); Benoit and Gagnaire-Renou (2007).

\subsection{Directional spreading of swell spectra}

Despite a significant difference in the runs in integral characteristics of the swell anisotropy (e.g., Fig. 2b, d), the resulting spectral distributions still show pronounced features of universality, as is seen in frequency spectra (Fig. 4). As will be shown below, this universality of swell spectra is seen in angular distributions as well. This is of importance in the context of remarks of Sect. 2.2: while the shape functions $\Phi_{p_{\tau}}, \Phi_{p_{\chi}}$ of self-similar solutions (Eqs. 8 and 9) are not unique, there is likely a mechanism of their selection that supports the universality of the swell spectral distributions. Within a linear theory, it could be treated as survival of the only eigenfunction or, more prudently, of very few eigenmodes of the problem. As mentioned in Sect. 2.2. this "linear" treatment can be used with some reservations for our problem, which is heavily nonlinear in terms of wave spectra but allows for a quasi-linear analysis in terms of spectral fluxes (see Zakharov and Pushkarev, 1999; Pushkarev et al., 2003).

The only physical mechanism of the mode selection in the swell problem is nonlinear relaxation to an inherent state due to four-wave resonant interactions. This relaxation generally occurs at essentially shorter timescales than ones of wind pumping and wave dissipation (Zakharov and Badulin, 2011). There is no contradiction with the present vision of the sea wave balance in the above statement. The effect of nonlinear interactions on wave spectra is 2-fold: firstly, it supports an inherent shaping of the spectra by very fast feedback to its perturbation and, secondly, it is responsible for relatively slow nonlinear cascading within this inherent shaping.

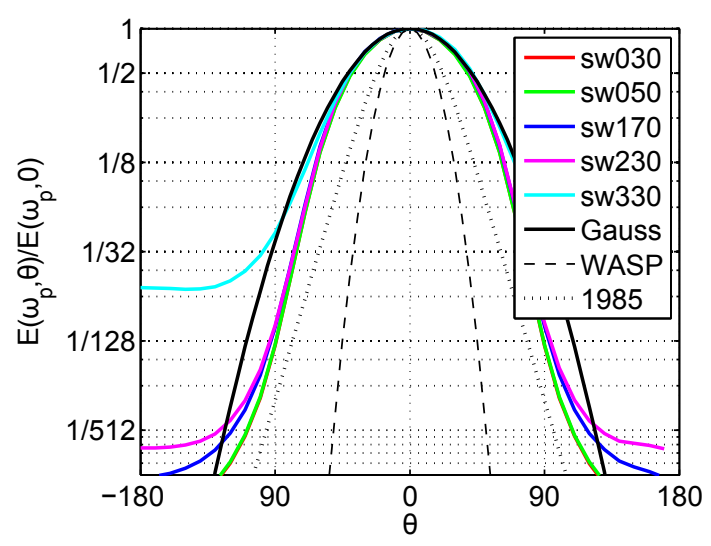

Figure 5. Normalized dependence of swell energy spectra on angle at peak frequency $\omega_{\mathrm{p}}$ after 11.5 days (approximately $10^{6} \mathrm{~s}$ ) of swell evolution for the runs of Table 1 (see the legend). Dashed line - Gaussian distribution (Eq. 29) with dispersion $\sigma_{\Theta}=35^{\circ}$; dotted line - growing sea (Eq. 30) and Eq. 9.1-9.2 of Donelan et al. (1985); dashed line - wrapped-normal fit of Ewans et al. (2004, Table 11.2, Fig. 11.8).

Normalized sections of spectra at the peak frequency $\omega_{\mathrm{p}}$ are shown in Fig. 5 for runs of Table 1 at $t=10^{6} \mathrm{~s}$ (approx. 11.5 days). "The almost isotropic" run sw330 shows a relatively high pedestal of about $2 \%$ of the maximal value, while other series have a background of 1 order less. At the same time, the core of all distributions is quite close to a Gaussian shape

$y_{\text {gauss }}=\exp \left(-\frac{\theta^{2}}{2 \sigma_{\Theta}^{2}}\right)$

with half-width $\sigma_{\Theta}=35^{\circ}$ (dashed curve in Fig. 5). Experimentally based spreading functions are represented in Fig. 5 by two reference curves. For growing wind seas the dependence by Donelan et al. (1985, Eq. 9.2)

$y_{1985}=\operatorname{sech}^{2}(\beta \theta) ; \quad \beta=2.28$

gives almost twice as narrow a distribution (dotted line in Fig. 5). The wrapped-normal fit of angular distribution for one of the case of the West Africa Swell Project (see Table 11.2 and Fig. 11.8 in Ewans et al., 2004) with standard deviation $\sigma_{\Theta} \approx 14.3^{\circ}$ gives a sharper distribution shown by a dashed curve.

Evolution of directional spreading in time is shown in absolute values in Fig. 6 for three runs: the most anisotropic case sw030 (Fig. 6a, b), weakly anisotropic initial state sw230 (Fig. 6c, d) and "the almost isotropic" run sw330 (Fig. 6e, f). In the left column the angular spreading at peak frequency shows remarkably close patterns for the first two cases: peak values at large times differ by a few percent only. The weakly anisotropic case sw230 (initial angular spreading $230^{\circ}$ with essential counter-propagating fraction) reaches 

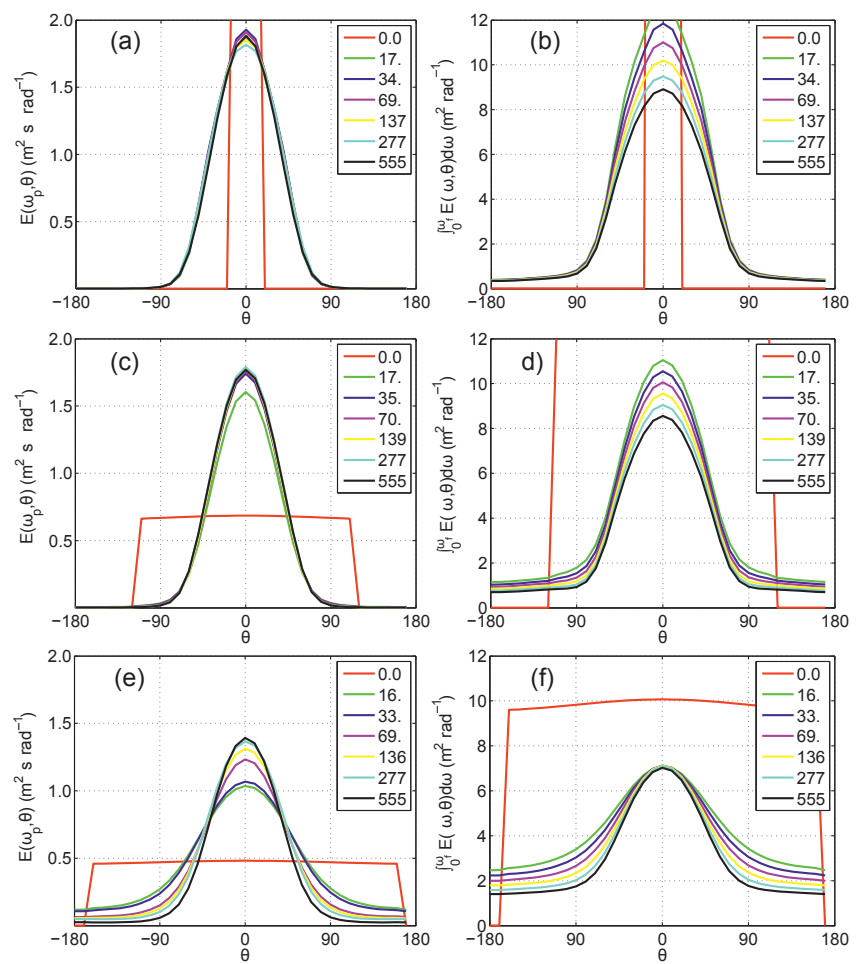

Figure 6. Angular spreading of the swell spectra at different times (in hours; see the legend). Left column - wave spectra at peak frequency; right - integral of wave spectra in frequency as a function of direction. (a, b) run sw030 of Table 1 - strong initial anisotropy; (c, d) run sw230 - weak anisotropy; (e, f) "almost anisotropic" run sw330.

its almost saturated state for a couple of days only (cf. the curves at $t=17 \mathrm{~h}$ and $t=35 \mathrm{~h}$ ). Similar proximity of these two cases can be observed for integrals of spectra in frequency as shown in the right column of Fig. 6, i.e., for values

$\mathcal{E}(\theta)=\int_{0}^{\omega_{c}} E(\omega, \theta) \mathrm{d} \omega$.

Self-similar solutions (Eq. 8) predict a power-law decay of magnitude of $\mathcal{E}$ with time which is what we see in Fig. 6b, d for the first two cases. The behavior of "the almost isotropic" case sw330 is qualitatively different. The relatively strong adjustment to a narrow directional spreading occurs in the course of the entire duration $2 \times 10^{6} \mathrm{~s}$. The duration appears to be too short to reach a self-similar regime resembling cases sw030 and sw230.

The effect of sharpening of angular distributions of the run sw330 in Fig. 6e, f requires additional comments. First, it manifests a transitional nature of the case sw330 when a solution is rather far from its self-similar asymptotics. Secondly, this case illustrates the above statement of the paragraph on two scales of wave spectra evolution. The angular adjustment occurs at relatively short temporal scales as compared with
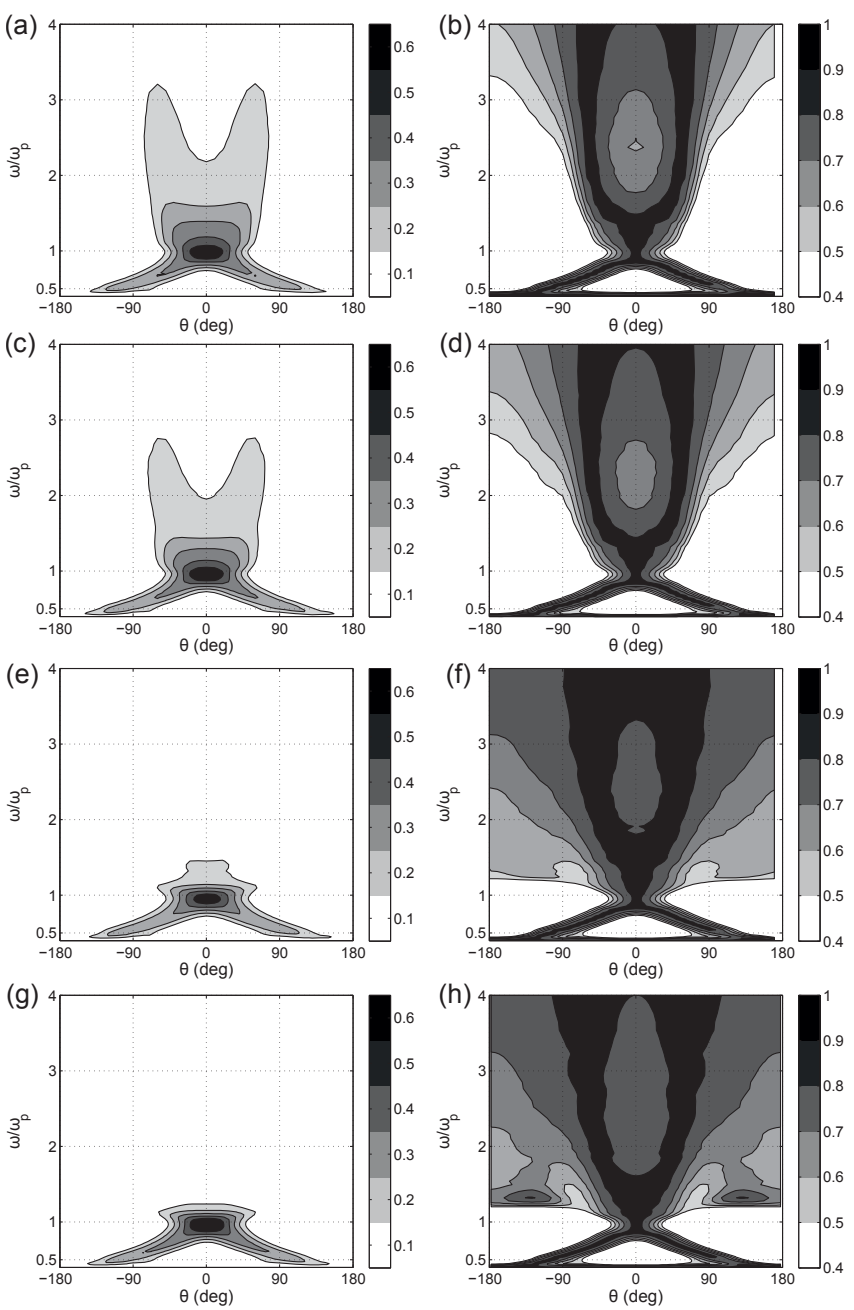

Figure 7. Isolines of spreading functions for different runs (see Table 1): (a, b) sw030; (c, d) sw170; (e, f) sw330; (g, h) run sw330 with finer resolution at angle $\Delta \theta=5^{\circ}$. Left column - definition (Eq. 32); right - directional distribution (Eq. 33).

slow evolution of integral parameters (cf. Fig. 2). This adjustment is provoked by excursion of initially "almost isotropic" distribution from an anticipated "inherent state" that, thus, stimulates wave-wave interactions as a mechanism of relaxation. The example demonstrates ability of wave-wave interactions to effectively rebuild directional distributions. Note that in some cases, say, in the problem of relaxation of wave field to sudden changes of wind direction the wave-wave interactions are considered ineffective as compared to relaxation "due mainly to imbalance $S_{\text {in }}<S_{\text {diss" }}$ " (e.g., Young et al., 1987, $S_{\text {in }}$ - wind input, $S_{\text {diss }}$ - wave dissipation).

\subsection{Bi-modality of swell spectra}

Bi-modality of directional spreading of ocean swell is widely discussed for experimental data as a possible result of swell evolution (e.g., Ewans, 1998, 2001; Ewans et al., 2004). Our 
simulations encounter this effect as a persistent feature of swell spectra. Figure 7 represents directional spreading of swell spectra in two ways. The left column shows directional distribution function $H(\omega, \theta)$ in the spirit of widely used definition (e.g., Ewans, 1998)

$$
\begin{gathered}
E(\omega, \theta)=\bar{E}(\omega) H(\omega, \theta), \quad H(\omega, \theta) \geq 0, \\
\int_{-\pi}^{\pi} H(\omega, \theta) \mathrm{d} \omega=1 .
\end{gathered}
$$

An alternative representation in the right column of Fig. 7 uses spectral densities normalized by their maxima at a fixed frequency to trace "ridges" of the surface $\widetilde{E}(\omega, \theta)$ defined as follows (cf. Eq. 1 in Young et al., 1995):

$\widetilde{E}(\omega, \theta)=E(\omega, \theta) / \max _{-\pi<\theta \leq \pi}(E(\omega, \theta))$.

Both representations reveal bi-modality of swell spectra fairly well for all cases of Table 1. "Narrow" initial spectrum sw030 and "wide" one sw170 evolve to very close X-shaped side-lobe patterns (Fig. 7a, c). Pronounced side lobes are seen both above and below the spectral peak frequency. The directional distribution function $H(\omega, \theta)$ (Eq. 32) does not show a similar pattern for "the almost isotropic" case sw330 (Fig. 7e, $\mathrm{g}$ ), but the X-shapes are seen fairly well in the "ridge" representation (Eq. 33) for all the cases. Directional spreading for run sw330 is shown for simulations with standard angular resolution $\Delta \theta=10^{\circ}$ (Fig. 7e, f) and with fine one $\Delta \theta=5^{\circ}$ (Fig. $7 \mathrm{~g}, \mathrm{~h}$ ). Higher resolution makes "ridges" sharper and allows for resolving of more details of the directional distribution. In particular, side lobes appear for counter-propagating waves at $\theta \approx \pm 3 \pi / 4$ and $\omega / \omega_{\mathrm{p}} \approx 5 / 4$. At the same time, the standard angular resolution in our simulations $\Delta \theta=10^{\circ}$ seems to be adequate for the bi-modality phenomenon.

The patterns similar to ones of Fig. 7 have been obtained in simulations of the Hasselmann equation for wind-driven waves with the exact term of nonlinear transfer $S_{\mathrm{nl}}$ by Banner and Young (1994); Young et al. (1995) at formally finer resolution $\Delta \theta=6.67^{\circ}$. It should be noted that directions beyond the cone $\theta= \pm 120^{\circ}$ have not been taken into account to speed up calculations in the cited papers. It can explain the discrepancy with our results at the high-frequency end of Fig. 7f, h (cf. Plate 1 in Young et al., 1995). This point can be clarified in further studies.

An important issue of agreement of our results and findings by Banner and Young (1994) and Young et al. (1995) is the presence of low-frequency (below the spectral peak) side lobes. Experimental results by Ewans (cf. Figs. 8 and 16; 1998) show good correspondence of the directional spreading functions to numerical results at high frequencies, but do not fix any side lobes below the spectral peak.

Generally, the phenomenon of side-lobe occurrence is associated with a joint effect of wave-wave interactions and wave generation by wind (e.g., Banner and Young, 1994;
Pushkarev et al., 2003; Bottema and van Vledder, 2008). The theoretical background of Sect. 2.1 and our simulations of swell can propose an interpretation and alternative ways of advanced analysis of the effect in terms of stationary solutions of Kolmogorov-Zakharov (Eq. 7). These solutions being presented as power series of dimensionless ratios of spectral fluxes and as an extension of the approximate solution Eq. (6) by Kats and Kontorovich (1974) predict higher-order angular harmonics and can be found within the formal procedure of Pushkarev et al. (2003, 2004). This approach is not fully correct in the vicinity of the spectral peak, but still looks plausible and useful for interpretation of the effect of wavewave interactions. Analysis of the next paragraph shows perspectives of the KZ solution paradigm.

\subsection{Swell spectra vs. KZ solutions}

The very slow evolution of swell in our simulations provides a chance to check the relevance of the classic KolmogorovZakharov solutions (Eqs. 4-7) to the problem under study. The key feature of the swell solution from the theoretical viewpoint is its "hybrid" (in the words of Badulin et al., 2005a) nature: the inverse cascade (negative fluxes) determines the evolution of the spectral peak and its downshifting, while the direct cascade (positive fluxes) occurs at frequencies slightly (approximately $20 \%$ ) above the peak. This hybrid nature is illustrated by Fig. 8 for energy and wave momentum fluxes. In order to avoid ambiguity in the treatment of the simulation results within the weak turbulence theory, we will not discuss this hybrid nature of swell solutions, and will focus on the direct cascade regime. Thus, the general solution (Eq. 7) in the form

$E(\omega, \theta)==\frac{P^{1 / 3} g^{4 / 3}}{\omega^{4}} G(0, g M /(\omega P), \theta)$

and its approximate explicit version Eq. (6) by Kats and Kontorovich $(1971,1974)$ will be used below for describing the direct cascading of energy and momentum at high frequency (as compared to $\omega_{\mathrm{p}}$ ).

Two runs of Table 1, sw030 and "almost isotropic" sw330, are presented in Fig. 8 in order to show qualitative similarity of extreme cases of initial directional spreading. Positive fluxes $P$ and $M$ decay with time, in good agreement with power-law dependencies (Eq. 22), and have rather low variations in a relatively wide frequency range $3 \omega_{\mathrm{p}}<\omega<6 \omega_{\mathrm{p}}$ in Fig. 8. For energy flux $P$ (Fig. 8a, b) one can see good quantitative correspondence (note that the times for some curves are slightly different). Absolute values of momentum flux $M$ as well as magnitudes of wave momentum itself (see Fig. 2) differ by more than 1 order.

The domain of quasi-constant fluxes $\omega>3 \omega_{\mathrm{p}}$ can be used for verification of the relevance of the stationary KZ solutions (Eqs. 4-6) to the quasi-stationary swell solutions. All the cases of Table 1 show very close patterns of spectral 

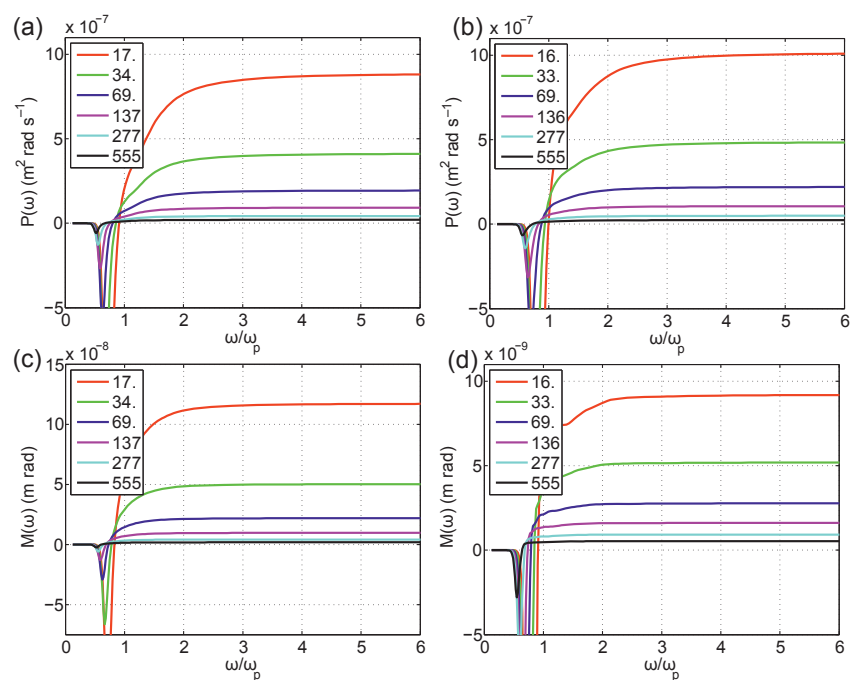

Figure 8. Top row - spectral fluxes of energy for series sw030 (a) and sw330 (b); bottom row - spectral fluxes of momentum for series sw030 (c) and sw330 (d) at different times (legend, in hours).

fluxes (e.g., Fig. 8) and, what is more important, very close estimates of Kolmogorov's constants.

The first and second Kolmogorov constants can easily be estimated for the approximate solution (Eq. 6) from combinations of along- and counter-propagating spectral densities as follows:

$C_{p}=\frac{\omega^{4}(E(\omega, 0)+E(\omega, \pi))}{4 g^{4 / 3} P^{1 / 3}}$,

$C_{m}=\frac{\omega^{5} P^{2 / 3}(E(\omega, 0)-E(\omega, \pi))}{4 g^{7 / 3} M}$.

These estimates provide very close values of the Kolmogorov constants for all the series of Table 1 with the only exception of "the almost isotropic" run sw330 for the second Kolmogorov constant $C_{m}$. Fig. 9 gives the first Kolmogorov constant $C_{p} \approx 0.21 \pm 0.01$ (slightly lower values for initially narrow distributions) and $C_{m} \approx 0.08 \pm 0.02$ for all the runs except sw330 (cf. Figs.9b, d for "narrow" sw030 and "wide" sw230).

The analytic estimate gives the very close result $C_{p}=$ 0.219 (Zakharov, 2010, Eq. 4.33). Numerical simulations by Lavrenov et al. (2002), Pushkarev et al. (2003), and Badulin et al. (2005a) missed a factor of 2 in definitions of the Kolmogorov constants (cf. our definitions in Eqs. 46 and Eqs. 4.29 and 4.30 in Zakharov, 2010). Taking this into account, one has the reported values $0.151<C_{p}<$ $0.162 ; 0.105<C_{m}<0.121$ in Lavrenov et al. (2002, Table 1), $0.16<C_{p}<0.23 ; 0.09<C_{m}<0.14$ in Pushkarev et al. (2003, eqs. 5.3, 5.6, 5.8) and $0.19<C_{p}<0.20$ in Badulin et al. (2005a). The first experimental attempt to evaluate the first Kolmogorov constant by Deike et al. (2014) presented the value $C=1.8 \pm 0.2 \approx 2 \pi C_{p}$, i.e., a $2 \pi$ times bigger counterpart of $C_{p}$.
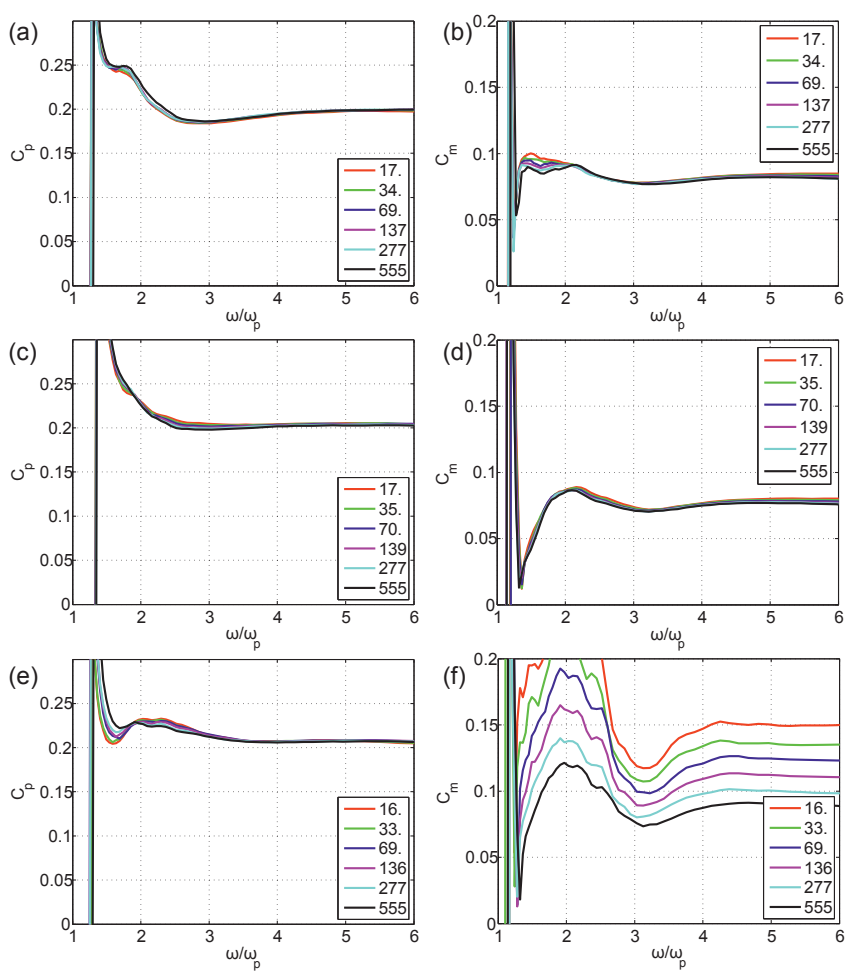

Figure 9. Left - estimates of the first Kolmogorov constant $C_{p}$; right - estimates of the second Kolmogorov constant $C_{m}$ for the approximate anisotropic KZ solution (Eq. 6). (a, b) run sw030; (c, d) sw230; (e, f) sw330. Time in hours is given in the legend.

While the estimates of the Kolmogorov's constants for the swell look consistent, the numerical solutions differ essentially from the approximate weakly anisotropic $\mathrm{KZ}$ solution (Eq. 6). The directional spreading cannot be described by the only angular harmonics as in Eq. (6); higher-order corrections are clearly seen in Fig. 7 as side lobes. Nevertheless, the robustness of the estimates of the second Kolmogorov constant $C_{m}$ provides a good reference for estimates of the spectra anisotropy.

The estimates of $C_{m}$ for sw330 (Fig. 9f) demonstrate a specific nonstationarity of the swell solution in terms of wave momentum flux, while the first Kolmogorov constant $C_{p}$ (Fig. 9f) shows the relevance of the stationary KZ solutions to the swell problem.

\section{Discussion. Swell and the ocean environment}

Results of our simulations showed their fairly good correspondence to findings of the theory of wave (weak) turbulence. The relevance of these results to experimental facts seems to be a logical conclusion of this work. The issue of relevance is 2 -fold. First, our results can help in explaining effects whose interpretation in terms of alternative approaches (mostly, within linear theory) is questionable. Sec- 

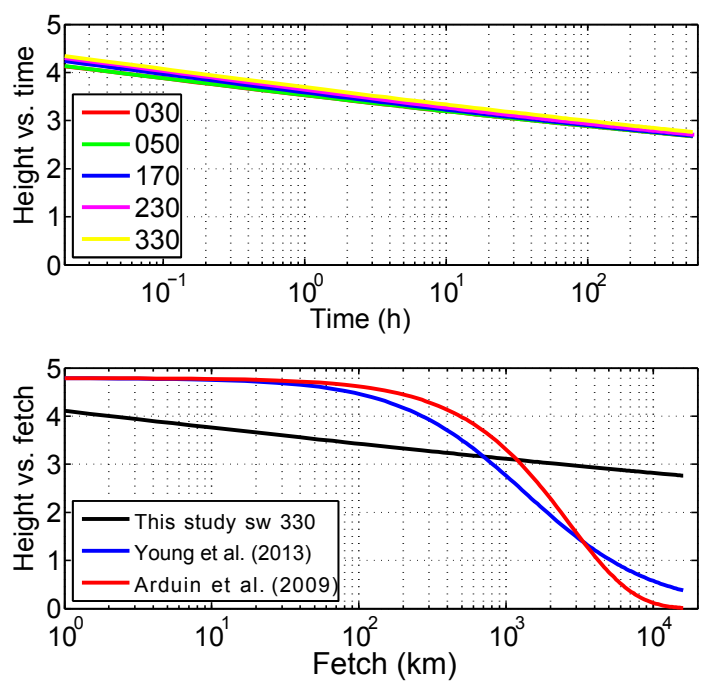

Figure 10. Top - dependence of significant wave height $H_{S}$ on time for the cases of Table 1. Bottom - attenuation of swell for models Ardhuin et al. (2009); Young et al. (2013) and one of this paper (see the legend). Results of duration-limited simulations are recasted into dependencies on fetch by simple transformation (Eq. 36).

ondly, one can formulate, or, at least, sketch cases where our approach becomes invalid or requires an extension. Both aspects are considered in the final section.

Attenuation in course of long-term swell evolution is an appealing problem of the swell monitoring. We show that contribution of wave-wave interactions to this process can be important mostly at initial stages of swell evolution. The observed rates of swell attenuation in an open ocean cannot be treated within our approach for a number of reasons. First of all, the duration-limited setup of our simulations do not account for important mechanisms of frequency dispersion and spatial divergence due to sphericity of the Earth. These mechanisms can both contribute into swell attenuation together with wave-wave interactions and essentially contaminate results of observations. The intrinsic swell attenuation is, generally, small as compared to the effect of reduction (or amplification at large fetches) (see Fig. 2b in Ardhuin et al., 2009) which is accounted for within the linear model of geometrical optics whose validity is generally assumed for ocean swell.

Ocean swell for long times (fetches) likely becomes an important constituent of the ocean environment which can be heavily affected by relatively short wind-driven waves. We discuss the effect of swell amplification at rather low wind speeds and give tentative estimates based on the approach of this paper.

\subsection{Swell attenuation within the kinetic equation}

Dependence of wave height on time is shown in upper panel of Fig. 10 (see also Fig. 2) for the runs of Table 1. All the runs show quantitatively close evolution. Strong drop of up to $30 \%$ of initial value occurs within a relatively short time of about 1 day. An essential part of the wave energy leakage corresponds to this transitional stage at the very beginning of swell evolution when swell tends very rapidly to self-similar asymptotics. Afterwards, the decay becomes much slower following the power-law dependence of the self-similar solutions (Eq. 22).

For comparison with other models, and available observations, the duration-limited simulations have been recasted into dependencies of fetch through the simplest time-to-fetch transformation (e.g., Hwang and Wang, 2004; Hwang, 2006):

$x(s)=\int_{0}^{s} C_{g}\left(\omega_{\mathrm{p}}(t)\right) \mathrm{d} t$.

The equivalent fetch is estimated as a distance covered by a wave guide travelling with the group velocity of the spectral peak component. The corresponding dependencies are shown in bottom panel in Fig. 10. Two quasi-linear models by Ardhuin et al. (2009) and Babanin (2006) predict relatively slow attenuation at fetches in a "near zone" less than $1000 \mathrm{~km}$ (approximately $1 \mathrm{day}$ ) and then gradual decay up to very few of the percentage points of initial value at final distances about $18000 \mathrm{~km}$ where our model shows qualitatively different weak attenuation.

It should be noted that our model describes attenuation of the ocean swell "on its own" due to wave-wave interactions without any external effects. Thus, the effect of an abrupt drop in wave amplitude at short times (fetch) should be taken into consideration above all others when discussing the possible application of our results to swell observations and physical interpretation of the experimental results.

\subsection{Swell and wind-sea coupling. Arrest of weakly turbulent cascading}

Extremely weak attenuation of swell due to wave-wave interactions provokes a question on robustness of this effect. A variety of physical mechanisms in the ocean environment can change the swell evolution qualitatively. The above discussion of swell attenuation presents a remarkable example of such transformation when dissipation becomes dominant. Tracking of swell events from space gives an alternative scenario of transformation when swell appears to be growing. Satellite tracks can comprise up to $30 \%$ of cases of growing swell; "most of them are not statistically significant" (Jiang et al., 2016). Nevertheless, a possible effect of wind-sea background on long ocean swell opens an important discussion in view of theoretical (Badulin et al., 2008b) and experimental (Benilov et al., 1974; Badulin and Grigorieva, 2012) results that demonstrate swell amplification by a wind-wave background.

As noted and shown above, evolution of swell can occur at different timescales for different physical quantities. 
Integrals of motion (energy, action, momentum) evolve at relatively large scales: frequency downshift and energy follows power-law dependencies $1 / 11\left(\omega_{\mathrm{p}} \sim t^{-1 / 11}\right.$ and $E \sim$ $\left.t^{-1 / 11}\right)$. The slow evolution is supported by interactions within a wave spectra that is close to an "inherent" quasistationary state.

Oppositely, spectral shaping is evolving due to excursions from an "inherent state" at much shorter scales that can be estimated following Zakharov and Badulin (2011, see Eqs. 21 and 22 therein). The nonlinear relaxation rate as defined by eqs. 14-16 of the cited paper can be written as

$\Gamma(\omega)=B \omega\left(\frac{\omega}{\omega_{\mathrm{p}}}\right)^{3} \mu^{4} H(\omega, \theta)$.

Here $B$ is a big dimensionless coefficient (e.g., $B=22.5 \pi \approx$ 70.7 for an isotropic spectrum; see Zakharov and Badulin, 2011) and $H(\omega, \theta)$ is the directional distribution function (Eq. 32). The big coefficient $B$ in (37) provides relatively fast relaxation of local excursions (in wave scales) from the slowly evolving "inherent" swell, especially, in the highfrequency domain (factor $\left(\omega / \omega_{\mathrm{p}}\right)^{3}$ in Eq. 37). Evidence of this relaxation can be seen in the evolution of the angular distribution of run sw330, where visible transformation of angular distribution is observed for the entire duration of more than 3 weeks (Fig. 6): the non-self-similar background of the swell spectra is feeding the core of the spectral distribution.

A similar effect can be realized in the mixed sea when background of relatively short wind-driven waves feeds the swell. Total energy flux of the swell is decaying as rapidly as $\mathrm{d} E / \mathrm{d} t \sim t^{-12 / 11}$ and at sufficiently large time the associated direct cascading can be arrested by inverse cascading of wind-driven waves which fast relaxation to an "inherent" swell ensures the swell feeding. This mechanism has been analyzed numerically (Badulin et al., 2008b) and showed its remarkable efficiency.

Simple estimates of the possibility of the effect can be made in terms of balancing of two fluxes: direct cascade of swell and inverse cascade of wind-driven fraction. The swell energy leakage can be estimated from the weakly turbulent law (Badulin et al., 2007, eq.1.9) as follows:

$$
\left(\frac{\mathrm{d} E}{\mathrm{~d} t}\right)_{\text {direct }}=\frac{E^{3} \omega_{\mathrm{p}}^{9}}{\alpha_{\mathrm{swell}}^{3} g^{4}}=\frac{\mu_{\text {swell }}{ }^{6} C_{\mathrm{swell}}^{3}}{\alpha_{\text {swell }}^{3} g} .
$$

Here swell parameters are marked by proper subscripts: $C_{\text {swell }}=g / \omega_{\mathrm{p}}-$ phase velocity of the spectral peal component; $\mu_{\text {swell }}-$ swell steepness by definition (Eq. 15); and $\alpha_{\text {swell }}$ - self-similarity parameter ( $\alpha_{\mathrm{ss}}$ in Badulin et al., 2007). Similar conversion of sea state parameters to spectral flux can be done for the wind-sea fraction (see Sect. 5.1 in Badulin et al., 2007, or Table 1 in Gagnaire-Renou et al., 2011):

$$
\left(\frac{\mathrm{d} E}{\mathrm{~d} t}\right)_{\text {inverse }} \approx C_{w}\left(\frac{\rho_{a}}{\rho_{w}}\right)^{3} \frac{U_{10}^{3}}{\alpha_{\text {wind }}^{3} g}
$$

where coefficient $C_{w}=O(1)$ is introduced as soon as the conversion is based on dimensonal analysis and generalization of experimental results (Toba, 1972). A counterpart of $\alpha_{\text {swell }}$, the self-similarity parameter $\alpha_{\text {wind }}$, is approximately 2 times less in magnitude (Badulin et al., 2007). Thus, the condition of the balance of fluxes assotiated with different fractions of the mixed sea (Eqs. 38, 39) says that

$2 C_{w} \frac{\rho_{a}}{\rho_{w}} \frac{U_{10}}{C_{\text {swell }}} \approx \mu_{\text {swell }}^{2}$.

For relatively short swell with period $T_{p}=10 \mathrm{~s}(\lambda \approx 150 \mathrm{~m})$ and wind speed $U_{10}=7 \mathrm{~m} \mathrm{~s}^{-1}$ one gets a critical swell steepness $\mu_{\text {swell }} \approx 0.03$. In other words, the mean-over-ocean wind $7 \mathrm{~m} \mathrm{~s}^{-1}$ can balance (arrest) direct cascading of rather steep swell and, hence, provoke a growth of the swell due to absorbing short wind-driven waves. Evidently, this simple balance model gives very tentative estimate of the effect. Nevertheless, visual observations (Badulin and Grigorieva, 2012) and satellite data (Jiang et al., 2016), in our opinion, provide telling arguments for this phenomenon. Thus, "negative dissipation" of swell (in the words of Jiang et al., 2016) could find its explanation within the simple model.

The simple estimate (Eq. 40) shows the limited value of our "pure swell" model for the ocean environment. Potentially, the effect of even light wind on long-term propagation of swell can change the result qualitatively. Our pilot numerical studies (see also Badulin et al., 2008b) show the importance of the swell and wind-sea coupling. This effect will be detailed in our further studies.

\section{Conclusions}

We presented results of sea swell simulations within the framework of the kinetic equation for water waves (the Hasselmann equation) and treated these properties within the paradigm of the theory of weak turbulence. A series of numerical experiments (duration-limited setup, WRT algorithm) has been carried out in order to outline features of wave spectra in a range of scales usually associated with ocean swell, i.e., wavelengths larger than $100 \mathrm{~m}$ and duration of propagation up to $2 \times 10^{6} \mathrm{~s}$ (more than 23 days). It should be stressed that the exact collision integral $S_{\mathrm{nl}}$ (nonlinear transfer term) has been used in all the calculations. Alternative, mostly operational approaches, like the DIA (discrete interaction approximation approach), can corrupt the results quantitatively and even qualitatively.

Key results of the study are the following.

1. A strong tendency for self-similar asymptotics is demonstrated. These asymptotics are shown to be insensitive to initial conditions in terms of evolution of integral quantities (wave energy, momentum). Moreover, universal angular distributions of wave spectra at large times have been obtained for both narrow (initial angular spreading $30^{\circ}$ ) and almost isotropic initial spectra. 
Bi-modality of the spectral distributions in our simulations is found to be in agreement with previous numerical and experimental results (Banner and Young, 1994; Ewans, 2001; Ewans et al., 2004). The universality of the spectral shaping can be treated as an effect of mode selection when very few eigenmodes of the boundary problem determine the system evolution. The inherent features of wave-wave interactions are responsible for this universality making the effect of initial conditions insignificant. Generally, the self-similar swell co-exists with a background, which is far from a self-similar state.

2. The classic Kolmogorov-Zakharov (KZ) isotropic and weakly anisotropic solutions for direct and inverse cascades are shown to be relevant to slowly evolving sea swell solutions. Estimates of the corresponding KZ constants are found to agree well with previous analytical, numerical and experimental results. Thus, features of $\mathrm{KZ}$ solutions can be used as a reference for advanced approaches in the swell studies.

3. We show that an inherent peculiarity of the Hasselmann equation, energy and momentum leakage, can also be considered a mechanism of the sea swell attenuation. Today's models of sea swell are unlikely to account for this effect. Possible problems of the models are sketched in Sect. 3.1 when different options of simulation of the "conservative dissipation" are discussed. All these options require sufficiently large high-frequency range where the short-term oscillations in absence of dissipation or hyper-viscosity can mimic the energy leakage at $|\boldsymbol{k}| \rightarrow \infty$. It should be noted that the energy decay rates of sea swell in the numerical experiments, generally, do not contradict the results of recent swell observations and modeling. These studies based on satellite data and wave model hindcasting are focused mostly on "far field" behavior of swell, generally, 1000 or more kilometers away from a stormy area. Our simulations show that a dramatic transformation of the swell occurs at shorter distances, in the "near field". The essential swell energy losses in the near field, mostly due to nonlinear transfer, is an intriguing challenge for sea wave forecasting since the very first discussions of the phenomenon within the concept of wave-wave interactions (e.g., Sect. 8a, b in Snodgrass et al., 1966). Thus, Fig. 10 outlines different domains of our model relevance rather than the model relevance for the general problem of ocean swell attenuation.

4. Long-term evolution of swell is associated with rather slow frequency downshift $\left(\omega_{\mathrm{p}} \sim t^{-1 / 11}\right)$ and energy attenuation $\left(E \sim t^{-1 / 11}\right)$. Meanwhile, the decay of other wave field quantities is essentially faster: wave steepness is decaying as $\mu \sim t^{-5 / 22}$, and total spectral flux is even faster: $\mathrm{d} E / \mathrm{d} t \sim t^{-12 / 11}$. This point is of key importance in our analysis as far as we consider non- linear cascades of wave energy as governing the physical mechanism of swell evolution. As we showed in the discussion, the weak direct cascade of swell can be arrested by relatively light winds and then swell can start to grow. In our opinion, this conclusion correlates with manifestations of swell amplification in satellite data (Jiang et al., 2016) and in visual observations (Badulin and Grigorieva, 2012). Thus, "negative dissipation" of swell (in the words of Jiang et al., 2016) could find its explanation within the simple estimate (Eq. 40) of Sect. 4.2.

5. The last conclusion uncovers deficiency of the durationlimited setup for the phenomenon of swell. An alternative setup of fetch-limited evolution $\left(\partial / \partial t \equiv 0, \nabla_{r} \neq 0\right)$ introduces dispersion of wave harmonics as a competing mechanism that can change the swell evolution dramatically. Recent advances in wave modeling (Pushkarev and Zakharov, 2016) make the problem of spatial-temporal swell evolution feasible and specify the perspectives of our first step study. The theoretical background for the classic fetch-limited setup when solutions depend on the only spatial coordinate (i.e., $\partial / \partial x \neq 0, \partial / \partial y \equiv 0)$ is sketched in Sect. 2 of this paper. The one-dimensional model adds an essential physical effect of wave dispersion. A passage to polar coordinates allows us to consider an effect of spatial divergence in formally one-dimensional problem where solutions depend on the radial coordinate but are still anisotropic in wavevector space. Self-similar solutions for this problem in the spirit of Sect. 2 can be easily found and related to numerical results. All the prospective simulations require developing effective numerical approaches. In particular, high angular resolution (not worse than $5^{\circ}$ ) could be recommended for these studies. V. Geogjaev and V. Zakharov has developed such code recently (a talk at the meeting Waves in Shallow Water Environment, 2016, Venice). We plan to use it in the swell studies.

Data availability. No data sets were used in this article.

Competing interests. The authors declare that they have no conflict of interest.

Acknowledgements. The authors are thankful for the support of Russian Science Foundation grant no. 14-22-00174. The authors are indebted to Victor Shrira and Vladimir Geogjaev for discussions and valuable comments. The authors are also grateful to Andrei Pushkarev for his assistance in simulations. The authors appreciate critical consideration of the paper by reviewers Gerbrant van Vledder and Sergei Annenkov. Their constructive feedback led to substantial revision of Sects. 3 and 4. 
Edited by: V. Shrira

Reviewed by: G. van Vledder, S. Y. Annenkov and two anonymous referees

\section{References}

Annenkov, S. Y. and Shrira, V. I.: Direct Numerical Simulation of Downshift and Inverse Cascade forWater Wave Turbulence, Phys. Rev. Lett., 96, 204501, https://doi.org/10.1103/PhysRevLett.96.204501, 2006.

Ardhuin, F., Chapron, B., and Collard, F.: Observation of swell dissipation across oceans, Geophys. Res. Lett., 36, L06607, https://doi.org/10.1029/2008GL037030, 2009.

Ardhuin, F., Rogers, E., Babanin, A. V., Filipot, J.-F., Magne, R., Roland, A., van der Westhuysen, A., Queffeulou, P., Aouf, J.-M. L. L., and Collard, F.: Semiempirical Dissipation Source Functions for Ocean Waves. Part I: Definition, Calibration, and Validation, J. Phys. Oceanogr., 40, 1917-1941, https://doi.org/10.1175/2010JPO4324.1, 2010.

Babanin, A. V.: On a wave-induced turbulence and a wave mixed upper ocean layer, Geophys. Res. Lett., 33, L20605, https://doi.org/10.1029/2006GL027308, 2006.

Badulin, S. I. and Grigorieva, V. G.: On discriminating swell and wind-driven seas in Voluntary Observing Ship data, J. Geophys. Res., 117, https://doi.org/10.1029/2012JC007937, 2012.

Badulin, S. I., Pushkarev, A. N., Resio, D., and Zakharov, V. E.: Direct and inverse cascade of energy, momentum and wave action in wind-driven sea, in: 7th International workshop on wave hindcasting and forecasting, 92-103, Banff, October 2002, available at: https://www.researchgate.net/publication/253354120_ Direct_and_inverse_cascades_of_energy_momentum_and_ wave_action_in_spectra_of_wind-driven_waves (last access: 20 May 2017), 2002.

Badulin, S. I., Pushkarev, A. N., Resio, D., and Zakharov, V. E.: Self-similarity of wind-wave spectra. Numerical and theoretical studies, in: Rogue Waves 2004, edited by: Olagnon, M. and Prevosto, M., Editions IFREMER, Brest, 39, 205-214, 2004.

Badulin, S. I., Pushkarev, A. N., Resio, D., and Zakharov, V. E.: Self-similarity of wind-driven seas, Nonlin. Processes Geophys., 12, 891-945, https://doi.org/10.5194/npg-12-891-2005, 2005a.

Badulin, S. I., Pushkarev, A. N., Resio, D., and Zakharov, V. E.: Universality of wind-wave spectra and exponents of wind-wave growth, in: Geophysical Research Abstracts, 7, 01515 pp., EGU, $2005 b$.

Badulin, S. I., Babanin, A. V., Resio, D., and Zakharov, V.: Weakly turbulent laws of wind-wave growth, J. Fluid Mech., 591, 339378, 2007.

Badulin, S. I., Babanin, A. V., Resio, D., and Zakharov, V.: Numerical verification of weakly turbulent law of wind wave growth, in: IUTAM Symposium on Hamiltonian Dynamics, Vortex Structures, Turbulence, Proceedings of the IUTAM Symposium held in Moscow, 25-30 August, 2006, edited by: Borisov, A. V., Kozlov, V. V., Mamaev, I. S., and Sokolovskiy, M. A., vol. 6 of IUTAM Bookseries, Springer, 175-190, ISBN: 978-1-4020-67433, 2008a.

Badulin, S. I., Korotkevich, A. O., Resio, D., and Zakharov, V. E.: Wave-wave interactions in wind-driven mixed seas, in: Proceed- ings of the Rogue waves 2008 Workshop, IFREMER, Brest, France, 77-85, 2008b.

Badulin, S. I., Zakharov, V. E., and Pushkarev, A. N.: Simulation of wind wave growth with reference source functions, Geophysical Research Abstracts, 15, EGU2013-5284-1, 2013.

Balk, A. M.: On the Kolmogorov-Zakharov spectra of weak turbulence, Phys. D: Nonlin. Phenom., 139, 137-157, 2000.

Banner, M. L. and Young, I. R.: Modeling Spectral Dissipation in the Evolution of Wind Waves. Part I: Assessment of Existing Model Performance, J. Phys. Oceanogr., 24, 1550-1571, https://doi.org/10.1175/15200485(1994)024<1550:MSDITE>2.0.CO;2, 1994.

Barber, N. F. and Ursell, F.: The Generation and Propagation of Ocean Waves and Swell. I, Wave Periods and Velocities, Philos. T. R. Soc. Lond., 240, 527-560, 1948.

Barrenblatt, G. I.: Scaling, self-similarity, and intermediate asymptotics: Dimensional analysis and intermediate asymptotics, Plenum Press, New York/London, 1979.

Beal, R. C., Kudryavtsev, V. N., Thompson, D. R., Grodsky, S. A., Tilley, D. G., Dulov, V. A., and Graber, V. A.: The influence of the marine atmospheric boundary layer on ERS-1 synthetic aperture radar imagery of the Gulf Stream, J. Geophys. Res., 102, 57995814, 1997.

Benilov, A. Y., Kouznetsov, O. A., and Panin, G. N.: On the analysis of wind wave-induced disturbances in the atmospheric turbulent surface layer, Bound.-Lay. Meteorol., 6, 269-285, 1974.

Benoit, M. and Gagnaire-Renou, E.: Interactions vague-vague nonlinéaires et spectre d'équilibre pour les vagues de gravité en grande profondeur d'eau, in: Proc. 18th Congrès Français de Mécanique, Grenoble (France), 2007.

Bottema, M. and van Vledder, G. P.: Effective fetch and non-linear four-wave interactions during wave growth in slanting fetch conditions, Coastal Eng., 55, 261-275, 2008.

Bottema, M. and van Vledder, G. P.: A ten-year data set for fetchand depth-limited wave growth, Coastal Eng., 56, 703-725, 2009.

Chen, K. S., Chapron, B., and Ezraty, R.: A Global View of Swell and Wind Sea Climate in the Ocean by Satellite Altimeter and Scatterometer, J. Atmos. Ocean. Technol., 19, 1849-1859, 2002.

Deike, L., Miquel, B., Gutiérrez, P., Jamin, T., Semin, B., Berhanu, M., Falcon, E., and Bonnefoy, F.: Role of the basin boundary conditions in gravity wave turbulence, J. Fluid Mech., 781, 196225, https://doi.org/10.1017/jfm.2015.494, 2014.

Delpey, M. T., Ardhuin, F., Collard, F., and Chapron, B.: Spacetime structure of long ocean swell fields, J. Geophys. Res., 115, https://doi.org/10.1029/2009JC005885, 2010.

Donelan, M. A., Hamilton, J., and Hui, W. H.: Directional spectra of wind-generated waves, Philos. T. R. Soc. Lond., 315, 509-562, 1985.

Ewans, K., Forristall, G. Z., Prevosto, M. O. M., and Iseghem, S. V.: WASP West Africa Swell Project, Final report, Ifremer - Centre de Brest, Shell International Exploration and Production, B.V., 2004.

Ewans, K. C.: Observations of the Directional Spectrum of FetchLimited Waves, J. Phys. Oceanogr., 28, 495-512, 1998.

Ewans, K. C.: Directional spreading in ocean swell, in: The Fourth International Symposium on Ocean Wave Measurement and Analysis, ASCE, San Francisco, 2001. 
Gagnaire-Renou, E., Benoit, M., and Forget, P.: Ocean wave spectrum properties as derived from quasi-exact computations of nonlinear wave-wave interactions, J. Geophys. Res., 115, 21562202, https://doi.org/10.1029/2009JC005665, 2010.

Gagnaire-Renou, E., Benoit, M., and Badulin, S. I.: On weakly turbulent scaling of wind sea in simulations of fetch-limited growth, J. Fluid Mech., 669, 178-213, 2011.

Hasselmann, K.: On the nonlinear energy transfer in a gravity wave spectrum. Part 1. General theory, J. Fluid Mech., 12, 481-500, 1962.

Hasselmann, K., Ross, D. B., Müller, P., and Sell, W.: A parametric wave prediction model, J. Phys. Oceanogr., 6, 200-228, 1976.

Henderson, D. M. and Segur, H.: The role of dissipation in the evolution of ocean swell, J. Geophys. Res.-Oceans, 118, 5074-5091, https://doi.org/10.1002/jgrc.20324, 2013.

Hwang, P. A.: Duration and fetch-limited growth functions of wind-generated waves parameterized with three different scaling wind velocities, J. Geophys. Res., 111, 2156-2202, https://doi.org/10.1029/2005JC003180, 2006.

Hwang, P. A. and Wang, D. W.: Field measurements of durationlimited growth of wind-generated ocean surface waves at young stage of development, J. Phys. Oceanogr., 34, 2316-2326, 2004.

Jiang, H., Stopa, J. E., Wang, H., Husson, R., Mouche, A., Chapron, B., and Chen, G.: Tracking the attenuation and nonbreaking dissipation of swells using altimeters, J. Geophys. Res.-Oceans, 121, 2169-9291, https://doi.org/10.1002/2015JC011536, 2016.

Kahma, K. K. and Pettersson, H.: Wave growth in a narrow fetch geometry, Glob. Atmos. Ocean Syst., 2, 253-263, 1994.

Kantha, L.: A note on the decay rate of swell, OM, 11, 167-173, https://doi.org/10.1016/j.ocemod.2004.12.003, 2006.

Kats, A. V. and Kontorovich, V. M.: Drift stationary solutions in the weak turbulence theory, JETP Letters, 14, 265-267, 1971.

Kats, A. V. and Kontorovich, V. M.: Anisotropic turbulent distributions for waves with a non-decay dispersion law, Soviet Physics JETP, 38, 102-107, 1974.

Lavrenov, I., Resio, D., and Zakharov, V.: Numerical simulation of weak turbulent Kolmogorov spectrum in water surface waves, in: 7th International workshop on wave hindcasting and forecasting, 104-116, Banff, October 2002, available at: https://www.researchgate.net/publication/312210314_ Lavr_7th_Workshop (last access: 30 May 2017), 2002.

Munk, W. H., Miller, G. R., Snodgrass, F. E., and Barber, N. F.: Directional Recording of Swell from Distant Storms, Philos. T. R. Soc. Lond., 255, 505-584, 1963.

Onorato, M., Osborne, A., Serio, M., Resio, D., Pushkarev, A., Brandini, C., and Zakharov, V. E.: Freely Decaying Weak Turbulence for Sea Surface Gravity Waves, Phys. Rev. Lett., 89, 144501, https://doi.org/10.1103/PhysRevLett.89.144501, 2002.

Pettersson, H.: Wave growth in a narrow bay, $\mathrm{PhD}$ thesis, University of Helsinki, available at: http://ethesis.helsinki.fi/julkaisut/ mat/fysik/vk/pettersson/ (last access: 30 May 2017), [ISBN 95153-2589-7 (Paperback) ISBN 952-10-1767-8 (PDF)], 2004.

Phillips, O. M.: On the dynamics of unsteady gravity waves of finite amplitude, J. Fluid Mech., 9, 193-217, 1960.

Pushkarev, A. and Zakharov, V.: On nonlinearity implications and wind forcing in Hasselmann equation, ArXiv e-prints, 2015.

Pushkarev, A. and Zakharov, V.: Limited fetch revisited: Comparison of wind input terms, in surface wave modeling, Ocean Mod- ell., 103, 18-37, https://doi.org/10.1016/j.ocemod.2016.03.005, 2016.

Pushkarev, A. N., Resio, D., and Zakharov, V. E.: Weak turbulent approach to the wind-generated gravity sea waves, Phys. D: Nonlin. Phenom., 184, 29-63, 2003.

Pushkarev, A., Resio, D., and Zakharov, V.: Second generation diffusion model of interacting gravity waves on the surface of deep fluid, Nonlin. Processes Geophys., 11, 329-342, https://doi.org/10.5194/npg-11-329-2004, 2004.

Snodgrass, F. E., Groves, G. W., Hasselmann, K. F., Miller, G. R., Munk, W. H., and Powers, W. H.: Propagation of Ocean Swell across the Pacific, Philos. T. R. Soc. Lond., 259, 431-497, 1966.

Toba, Y.: Local balance in the air-sea boundary processes. Part I. On the growth process of wind waves, J. Oceanogr. Soc. Jpn., 28, 109-121, 1972.

Toffoli, A., Onorato, M., Bitner-Gregersen, E. M., and Monbaliu, J.: Development of a bimodal structure in ocean wave spectra, J. Geophys. Res., 115, 2156-2202, https://doi.org/10.1029/2009JC005495, 2010.

Tracy, B. and Resio, D.: Theory and calculation of the nonlinear energy transfer between sea waves in deep water, WES Rep. 11, US Army, Engineer Waterways Experiment Station, Vicksburg, MS, 1982.

Tsimring, L. S.: On the theory of swell decay under opposing wind, Meteorologiya i Gydrologiya, 6, 76-81, 1986 (in Russian).

Webb, D. J.: Non-linear transfers between sea waves, Deep-Sea Res., 25, 279-298, 1978.

Young, I. R.: Directional spectra of hurricane wind waves, J. Geophys. Res., 111, 2156-2202, https://doi.org/10.1029/2006JC003540, 2006.

Young, I. R., Hasselmann, S., and Hasselmann, K.: Computations of the response of a wave spectrum to a sudden change in wind direction, J. Phys. Oceanogr., 17, 1317-1338, 1987.

Young, I. R., Verhagen, L. A., and Banner, M. L.: A note on the bimodal directional spreading of fetch-limited wind waves, J. Geophys. Res., 100, 773-778, https://doi.org/10.1029/94JC02218, 1995.

Young, I. R., Babanin, A. V., and Zieger, S.: The Decay Rate of Ocean Swell Observed by Altimeter, J. Phys. Oceanogr., 43, 2322-2333, 2013.

Zakharov, V. E.: Statistical theory of gravity and capillary waves on the surface of a finite-depth fluid, Eur. J. Mech. B/Fluids, 18, 327-344, 1999.

Zakharov, V. E.: Theoretical interpretation of fetch limited winddrivensea observations, Nonlin. Processes Geophys., 12, 10111020, https://doi.org/10.5194/npg-12-1011-2005, 2005.

Zakharov, V. E.: Energy balance in a wind-driven sea, Phys. Scr., T142, 014052, https://doi.org/10.1088/00318949/2010/T142/014052, 2010.

Zakharov, V. E. and Badulin, S. I.: On Energy Balance in WindDriven Seas, Doklady Earth Sciences, 440, 1440-1444, 2011.

Zakharov, V. E. and Filonenko, N. N.: Energy spectrum for stochastic oscillations of the surface of a fluid, Soviet Phys. Dokl., 160, 1292-1295, 1966.

Zakharov, V. E. and Pushkarev, A. N.: Diffusion model of interacting gravity waves on the surface of deep fluid, Nonlin. Processes Geophys., 6, 1-10, https://doi.org/10.5194/npg-6-1-1999, 1999. 
Zakharov, V. E. and Zaslavsky, M. M.: Kinetic equation and Kolmogorov spectra in the weak-turbulence theory of wind waves, Izv. Atmos. Ocean. Phys., 18, 970-980, 1982.

Zakharov, V. E., Lvov, V. S., and Falkovich, G.: Kolmogorov spectra of turbulence, Part I, Springer, Berlin, 1992.

Zakharov, V. E., Korotkevich, A. O., Pushkarev, A. N., and Resio, D.: Coexistence of Weak and Strong Wave Turbulence in a Swell Propagation, Phys. Rev. Lett., 99, 164501, https://doi.org/10.1103/PhysRevLett.99.164501, 2007.
Zakharov, V. E., Badulin, S. I., Hwang, P. A., and Caulliez, G.: Universality of Sea Wave Growth and Its Physical Roots, J. Fluid Mech., 708, 503-535, https://doi.org/10.1017/jfm.2015.468, 2015.

Zaslavskii, M. M.: Nonlinear evolution of the spectrum of swell, Izv. Atmos. Ocean. Phys., 36, 253-260, 2000. 